\title{
Emodin induces apoptosis of lung cancer cells through ER stress and the TRIB3/NF-kB pathway
}

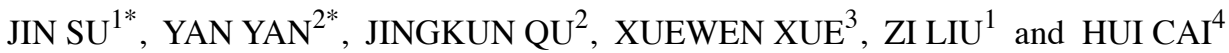 \\ ${ }^{1}$ Department of Radiotherapy, The First Affiliated Hospital of Xi'an Jiaotong University; ${ }^{2}$ The Second Department of Thoracic \\ Surgery, The First Affiliated Hospital of Xi'an Jiaotong University; ${ }^{3}$ Department of Surgery, Xi'an Feng Cheng Hospital; \\ ${ }^{4}$ Department of Vascular Surgery, The First Affiliated Hospital of Xi'an Jiaotong University, Xi'an, Shaanxi, P.R. China
}

Received August 14, 2016; Accepted January 2, 2017

DOI: $10.3892 /$ or.2017.5428

\begin{abstract}
Emodin is a phytochemical with potent anticancer activities against various human malignant cancer types, including lung cancer; however, the molecular mechanisms underlying the effects of emodin remain unclear. In the present study, the A549 and H1299 human non-small lung cancer cell lines were treated with emodin and the induced molecular effects were investigated. Changes in cell viability were evaluated by MTT assay, Hoechst staining was used to indicate the apoptotic cells, and western blotting was utilized to assess endoplasmic reticulum (ER) stress and signaling changes. RNA interference was also employed to further examine the role of tribbles homolog 3 (TRIB3) in the emodin-induced apoptosis of lung cancer cells. Emodin was found to reduce the viability of lung cancer cells and induce apoptosis in a concentrationdependent manner. Emodin-induced apoptosis was impaired by inhibition of ER stress using 4-phenylbutyrate (4-PBA). ER stress and TRIB3/nuclear factor- $\kappa \mathrm{B}$ signaling was activated in emodin-treated lung cancer cells. Emodin-induced apoptosis was reduced by TRIB3 knockdown in A549 cells, whereas ER stress was not reduced. In vivo assays verified the significance of these results, revealing that emodin inhibited lung cancer growth and that the inhibitory effects were reduced by inhibition of ER stress with 4-PBA. In conclusion, the results suggest that TRIB3 signaling is associated with emodin-induced ER stress-mediated apoptosis in lung cancer cells.
\end{abstract}

\section{Introduction}

Worldwide, and particularly in developing countries, lung cancer has become one of the leading causes of malignant

Correspondence to: Dr Hui Cai, Department of Vascular Surgery, The First Affiliated Hospital of Xi'an Jiaotong University, 277 West Yanta Road, Xi'an, Shaanxi 710061, P.R. China

E-mail: caihui9@stu.xjtu.edu.cn

*Contributed equally

Key words: lung cancer, emodin, endoplasmic reticulum stress, apoptosis, tribbles homolog 3 cancer-associated death due to its high rates of morbidity and mortality, and its poor prognosis (1). Non-small cell lung cancer (NSCLC) is the most common type of lung cancer, accounting for $80 \%$ of all lung cancer cases. The prognosis of NSCLC is poor, with a 5-year overall survival rate of only $\sim 15 \%$ (2). Surgery, chemotherapy and radiotherapy are the common treatment options for NSCLC. Surgery is considered the most effective treatment; however, many patients are not suitable for surgical intervention because of invasion and metastasis. Additionally, the adverse effects of radiotherapy and chemotherapy limit their clinical application (3). Therefore, searching for novel effective therapeutic agents and understanding their mechanism of action is of great clinical significance.

In recent decades, natural products extracted from herbs, termed phytochemicals, have attracted attention due to their reliable therapeutic effects and mild side effects (4). Emodin, also referred to as 1,3,8-trihydroxy-6-methyl-anthraquinone, is present in various Chinese medicinal herbs, including Rheum and Polygonum, which have been used since ancient times (5). Modern pharmacological and biomedical analyses have demonstrated the anticancer effects of emodin on several types of human cancer, including hepatic cancer (6), gallbladder cancer (7), cervical cancer (8), myelocytic leukemia (9) and lung cancer (10). However, our understanding of the molecular mechanisms of the effects of emodin as a potent anticancer agent on NSCLC cells is still inadequate to support the further clinical application of this phytochemical.

Tribbles homolog 3 (TRIB3) was initially identified as a pseudokinase that inhibits mitosis in the embryo and germ cells of Drosophila (11). Further studies detected the expression of TRIB3 in many human cell types and suggested that TRIB3 has important regulatory roles in cell apoptosis, autophagy and migration (12). It was reported that the expression of TRIB3 is induced when cells are under conditions of stress, including hypoxia and nutrient starvation (13). Activated endoplasmic reticulum (ER) stress in particular has been shown to trigger TRIB3 signaling activation, as transcription of TRIB3 was identified to be regulated by activating transcriptional factor 4 (ATF4) and $\mathrm{C} / \mathrm{BEP}$ homologous protein (CHOP) (14). A study in pancreatic $\beta$ cells showed that TRIB3 induced cell apoptosis via activation of nuclear factor- $\kappa \mathrm{B}(\mathrm{NF}-\kappa \mathrm{B})$ signaling (15). 
A recent study revealed that TRIB3 expression level was closely associated with the prognosis of malignant cancer, suggesting that TRIB3 is involved in cancer development and/or treatment resistance (16). Previous studies have confirmed that ER stress-induced cell death is one of the mechanisms underlying the anti-proliferative effects of emodin in malignant cancers (8). Thus, it is reasonable to hypothesize that TRIB3 may be a mediator of the ER stress-induced apoptosis caused by emodin in NSCLC cells.

In this study, the A549 and H1299 human NSCLC cell line were used to investigate the effects of emodin. After incubation with emodin, the proliferation and apoptosis of lung cancer cells were assessed. Markers of ER stress, including glucoseregulated protein 78 (GRP78) and CHOP were also detected. In addition, ER stress was inhibited using 4-phenylbutyrate (4-PBA) and RNA interference was used to silence TRIB3 expression. The results of the current study provide further knowledge of the anticancer activity of emodin and support for its future clinical application.

\section{Materials and methods}

Cell line and culture. A549 and H1299 human NSCLC cells were purchased from the China Center for Type Culture Collection (Wuhan, China). A549 cells were cultured in RPMI1640 medium (Gibco; Thermo Fisher Scientific, Inc., Waltham, MA, USA) while H1299 cells were cultured in RPMI-1640 (Gibco, Thermo Fisher Scientific, Inc.) supplemented with $10 \%$ fetal bovine serum (FBS; Hyclone; GE Healthcare, Logan, UT, USA), penicillin (100 U/ml; Sigma-Aldrich; Merck Millipore, Darmstadt, Germany), streptomycin (100 mg/ml; Sigma-Aldrich; Merck Millipore) and L-glutamine ( $2 \mathrm{mmol} / \mathrm{l}$; Sigma-Aldrich; Merck Millipore). Cells were cultured in an incubator with a humidified condition of $5 \% \mathrm{CO}_{2}$ and $95 \%$ air at $37^{\circ} \mathrm{C}$.

Small interfering RNA (siRNA) knockdown. siRNA was used to knockdown the expression of TRIB3 in A549 and H1299 cells, respectively. Cells were seeded in 6-well plates $\left(5 \times 10^{4}\right.$ cell/well) in $2 \mathrm{ml}$ media $24 \mathrm{~h}$ before transfection; cells were $80-90 \%$ confluent. The specific siRNA against TRIB3 (100 pmol/well, GenePharma, China) was transfected into A549 and H1299 cells with Lipofectamine 2000 reagent (Invitrogen; Thermo Fisher Scientific, Inc.), according to the manufacturer's instructions. After $48 \mathrm{~h}$ of transfection, cells were used for qRT-PCR or apopsis assay. The siRNA sequence used was as indicated in a previous study (15): 5'-ATC TCTGGCTGCTTCTGCCCATGTT-3'.

Cell grouping and treatment. Lung cancer cells were harvested in the logarithmic growth phase. Cells were incubated with serially diluted emodin (Selleck Chemicals, Shanghai, China) solutions $(0,20,40,60$ and $80 \mu \mathrm{mol} / \mathrm{l})$ for $48 \mathrm{~h}$. Equal numbers of cells were collected and divided into 6 different treatment groups as follows: Ctrl (control group); Ctrl + 4-PBA [treatment with 4-PBA (500 $\mu \mathrm{mol} / \mathrm{l})]$; Ctrl + siRNA (treatment with TRIB3 siRNA); emodin [treatment with emodin $(80 \mu \mathrm{mol} / \mathrm{l})$ ]; emodin + 4-PBA [co-treatment with emodin $(80 \mu \mathrm{mol} / \mathrm{l})$ and 4-PBA $(500 \mu \mathrm{mol} / \mathrm{l})$ ]; and emodin + siRNA [co-treatment with emodin $(80 \mu \mathrm{mol} / \mathrm{l})$ and TRIB3 siRNA].
Cell viability assessment. Colorimetric 3-(4,5-dimethylthiazol-2-yl) 2,5-diphenyltetrazolium bromide (MTT) assay (Sigma-Aldrich; Merck Millipore) was used to assess the viability of lung cancer cells in the afore-mentioned treatment groups. A549 and H1299 cells were seeded into 96-well plates and incubated with MTT $(5 \mathrm{mg} / \mathrm{ml})$ for $4 \mathrm{~h}$ at $37^{\circ} \mathrm{C}$. Subsequently, $150 \mu \mathrm{l}$ dimethylsulfoxide (Sigma-Aldrich; Merck Millipore) was added to the wells after washing with PBS. A plate reader (Bio-Rad Laboratories, Inc., Hercules, CA, USA) was used to detect the absorbance at $540 \mathrm{~nm}\left(\mathrm{~A}_{540}\right)$. The cell viability inhibition rate was calculated using the following formula: Viability inhibition $=\left[1-\mathrm{A}_{540}\right.$ (experimental well) / $\mathrm{A}_{540}$ (control well)] x100\%.

Cell apoptosis analysis. Hoechst fluorescent staining was used to indicate apoptotic cells. Briefly, cells were harvested and fixed with $4 \%$ paraformaldehyde at $37^{\circ} \mathrm{C}$ for $1 \mathrm{~h}$. Subsequently, the cells were washed with PBS and stained with $5 \mu \mathrm{mol} / 1$ Hoechst 33342 (Sigma-Aldrich; Merck Millipore) at $37^{\circ} \mathrm{C}$ for $30 \mathrm{~min}$ in a humidified dark chamber. The fluorescent images of Hoechst staining were captured using a fluorescence microscope (Cannon, Japan). Hoechst-positive cells were considered apoptotic.

Western blotting. Cell lysates of A549 and H1299 cells were collected after lysis in RIPA buffer (Santa Cruz Biotechnology, Inc., Dallas, TX, USA) according to the manufacturer's instructions. A protein extraction kit (Beyotime Institute of Biotechnology, Haimen, China) was used to extract the total protein, and a R0050 nuclear protein extraction kit (Beijing Solarbio Science \& Technology Co., Ltd., Beijing, China) was used to extract the nuclear proteins of lung cancer cells. Protein concentrations were assessed using a bicinchoninic acid kit (Thermo Fisher Scientific, Inc.). Vertical electrophoresis was applied to separate the proteins after loading in SDS gels, and the proteins were then electrotransferred onto nitrocellulose membranes (EMD Millipore, Billerica, MA, USA). After blocking with $5 \%$ non-fat milk, the membranes were incubated with specific antibodies against GRP78 (1:2,500; Abcam, Cambridge, MA, USA), CHOP (1:3,000; Abcam), TRIB3 (1:3,000; Cell Signaling Technology, Inc., Danvers, MA, USA),

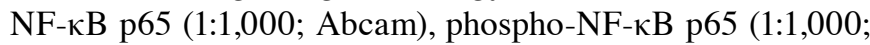
Cell Signaling Technology, Inc.), cleaved caspase-3 (1:2000, Abcam), pro-caspase3 (1:2000, Abcam), GAPDH (1:20,000; Santa Cruz Biotechnology, Inc.) and lamin B (1:250; Santa Cruz Biotechnology, Inc.). GAPDH was used as the internal control for total protein and lamin B as the internal control for nuclear proteins.

Tumor xenograft experiments. All in vivo experiments were approved by the Institutional Research Committee of Xi'an Jiaotong University (Xi'an, China). The mice received humane care in compliance with the Guide for the Care and Use of Laboratory Animals published by the National Institutes of Health. Cancer cells $\left(2.5 \times 10^{6}\right)$ were mixed in a $1: 1(\mathrm{v}: \mathrm{v})$ ratio with growth factor-reduced Matrigel (BD Biosciences, Franklin Lakes, NJ, USA), and the mixture was injected subcutaneously into the right flanks of 6- to 7-week-old $\mathrm{BALB} / \mathrm{c}$ nu/nu nude mice. At $24 \mathrm{~h}$ after implantation, mice bearing A549 cells were randomly assigned to 4 groups $(n=8$ 
A

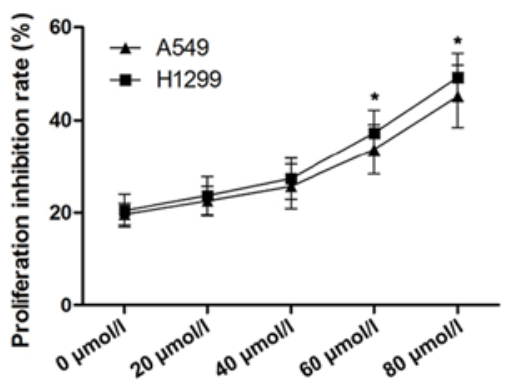

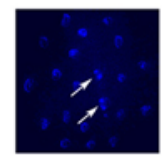
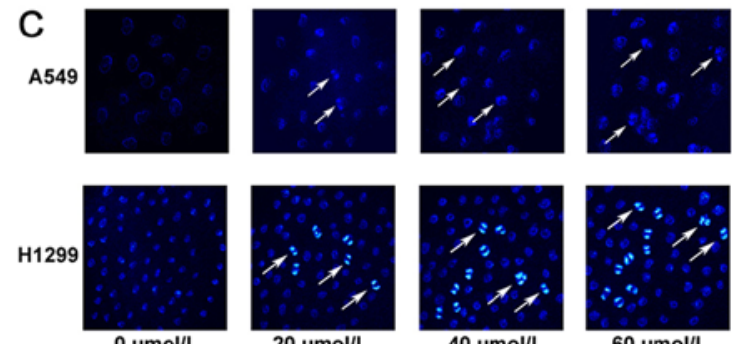

$60 \mu \mathrm{mol} / \mathrm{l}$

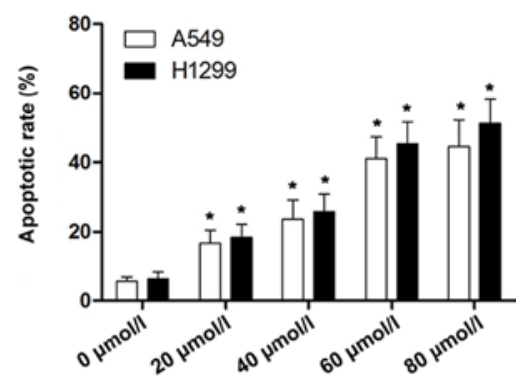

B<smiles>O=C(O)c1cc(O)c2c(c1)C(=O)c1cccc(O)c1C2=O</smiles>
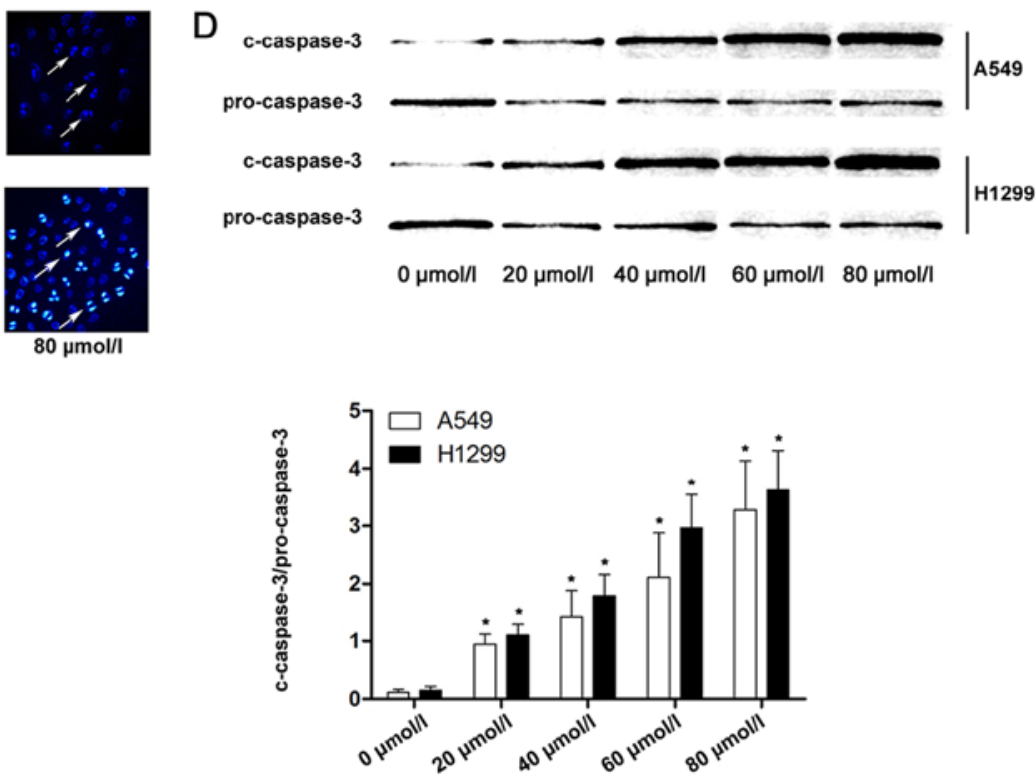

Figure 1. Emodin inhibits proliferation and induces apoptosis of A549 and H1299 cells in a concentration-dependent manner. (A) A549 and H1299 cells were incubated with emodin at $0,20,40,60$ and $80 \mu \mathrm{mol} / 1$ for $72 \mathrm{~h}$. The cell viability was detected by MTT assay and the proliferation inhibition rate was calculated. ${ }^{*} \mathrm{P}<0.05$. (B) The molecular structure of emodin. (C) A549 and H1299 cells were incubated with emodin at 0, 20, 40, 60 and $80 \mu \mathrm{mol} / 1$ for $72 \mathrm{~h}$. The apoptosis of A549 and H1299 cells was detected by Hoechst staining. The Hoechst-positive cells (white arrows pointed) were recognized as apoptotic. The lower panel presents the apoptotic rate of A549 and H1299 cells incubated with emodin at different concentrations according to the results of Hoechst staining. "P<0.05. (D) The apoptosis of A549 and H1299 cells was assessed by activation determination of caspase activation. The immunoblots of cleaved caspase-3 (c-caspase-3) and pro-caspase- 3 were demonstrated. Columns on the lower panel indicated the ratio of c-caspase-3 over pro-caspase-3 in lung cancer cells. " $\mathrm{P}<0.05$.

per group) as follows: i) Control; ii) $20 \mathrm{mg} / \mathrm{kg} / \mathrm{day} 4-\mathrm{PBA}$ alone; iii) $50 \mathrm{mg} / \mathrm{kg} / \mathrm{day}$ emodin; and iv) $50 \mathrm{mg} / \mathrm{kg} / \mathrm{day}$ emodin combined with $20 \mathrm{mg} / \mathrm{kg} / \mathrm{day} 4-\mathrm{PBA}$. Emodin and 4-PBA, suspended in saline, were intraperitoneally injected once per day. Controls received the vehicle alone $(20 \mathrm{ml} / \mathrm{kg})$. Tumor volume (measured in $\mathrm{mm}^{3}$ ) was determined using calipers every 2 days and calculated using the modified ellipse formula: Volume $=$ length $\mathrm{x}$ width $2 / 2$ (17). Mice were sacrificed by cervical dislocation on day 40. Implanted tumors were extracted and weighed.

Statistical analysis. Data acquired in this study is presented as the mean \pm standard deviation. Differences between groups were compared by analysis of variance and followed by Tukey's post-hoc tests. $\mathrm{P}<0.05$ was considered to indicate a statistically significant difference. All statistical analyses were processed by SPSS software (version 16.0; SPSS, Inc., Chicago, IL, USA).

\section{Results}

Emodin reduces viability and induces apoptosis of A549 cells in a concentration-dependent manner. We measured the viability of A549 and H1299 cells after incubation with emodin at 20,40,60 and $80 \mu \mathrm{mol} / 1$. The viability was significantly reduced at 60 and $80 \mu \mathrm{mol} / 1$ compared with the lower concentrations, as shown in Fig. 1A. Cell apoptosis is considered one of the most important mechanisms that mediates the proliferation-inhibitory effects of anticancer agents. The apoptosis of A549 and H1299 cells was assessed by Hoechst staining and caspase-3 activation. As shown in Fig. 1C and D, Hoechst-positive lung cancer cells were marked as apoptotic. Furthermore, as the concentration of emodin increased, the apoptotic rate and expression of cleaved caspase-3 of lung cancer cells were significantly increased compared with lower concentrations.

Apoptosis of lung cancer cells induced by emodin is mediated by ER stress. When exposed to harmful stimuli, prolonged ER stress causes cell death (18). GRP78 and CHOP were used as markers of ER stress in the current study. Furthermore, as a pro-apoptotic factor, CHOP is also closely associated with ER stress-induced cell death (19). 4-PBA has been applied as specific ER stress inhibitor in many previous studies (20). The current study demonstrated that the apoptosis induced by emodin was reduced significantly following 4-PBA treatment 

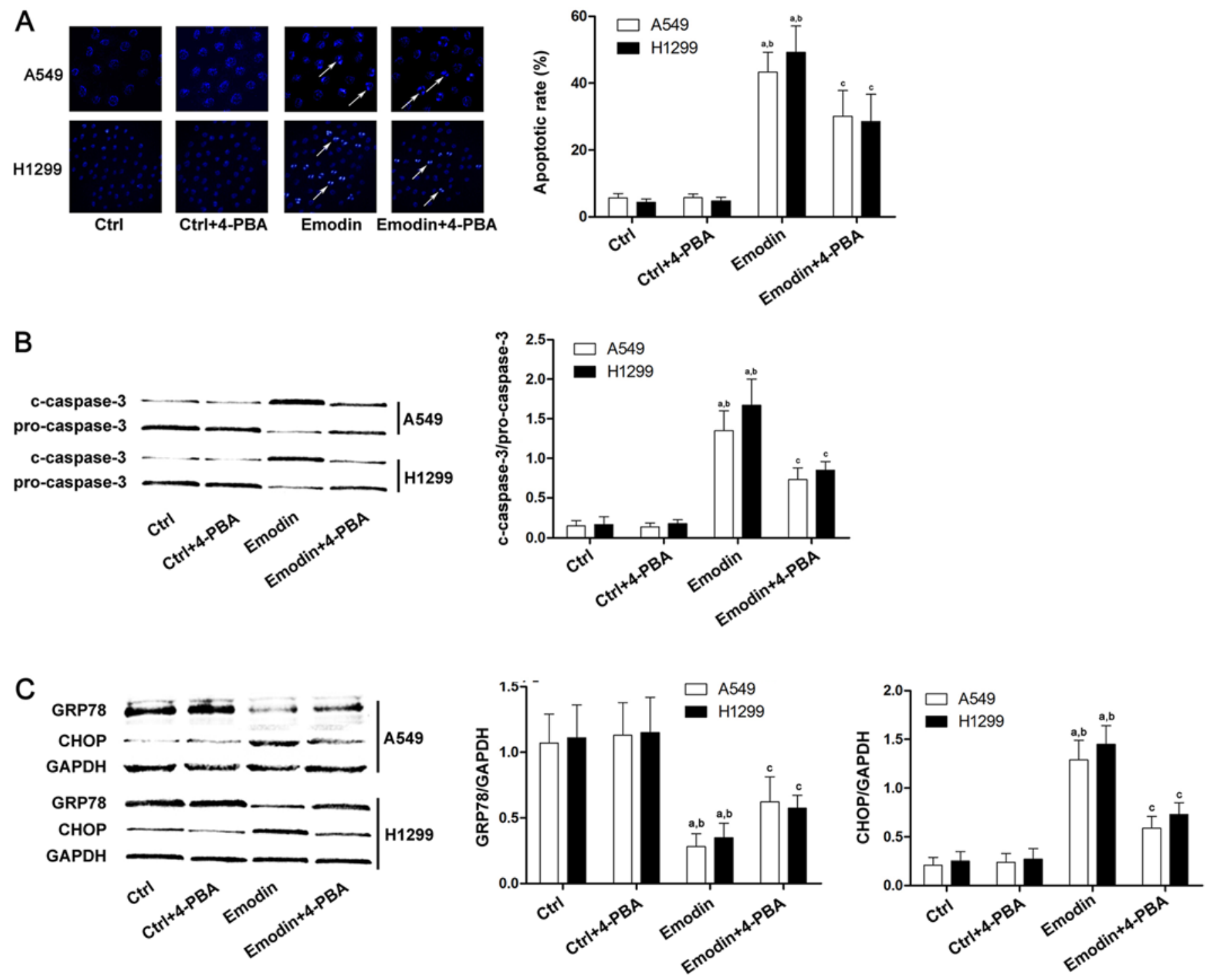

Figure 2. Apoptosis of A549 and H1299 cells induced by emodin is ER stress-mediated. Lung cancer cells were treated with emodin ( $80 \mu$ mol/1) with or without a specific ER stress inhibitor, 4-PBA $(500 \mu \mathrm{mol} / 1)$, for $72 \mathrm{~h}$. (A) The apoptosis of A549 and H1299 cells was detected by Hoechst staining. The white arrows indicate the Hoechst-positive cells. Columns demonstrated the apoptotic rates of A549 and H1299 cells in the Ctrl, Ctrl + 4-PBA, emodin, and emodin + 4-PBA groups. (B) Caspase-3 activation in different groups was detected by western blots. Columns on the right panel indicated the ratio of c-caspase- 3 over pro-caspase- 3 in lung cancer cells. The data are presented as the mean \pm standard deviation from three independent experiments. (C) Western blots of GRP-78, CHOP and GAPDH in the Ctrl, Ctrl + 4-PBA, emodin, and emodin + 4-PBA groups. GAPDH was used as the internal reference. Quantitation of western blots are presented as fold changes of GRP-78 and CHOP normalized to GAPDH. The data are presented as the mean \pm standard deviation from three independent experiments ( ${ }^{a} \mathrm{P}<0.05$ vs. Ctrl; ${ }^{\text {P }}<0.05$ vs. Ctrl + 4-PBA; ${ }^{\circ} \mathrm{P}<0.05$ vs. emodin). ER, endoplasmic reticulum; Ctrl, control; 4-PBA, 4-phenylbutyrate; GRP78, glucose regulating protein 78; $\mathrm{CHOP}, \mathrm{C} / \mathrm{BEP}$ homologous protein.

(Fig. 2A and B). Additionally, the expression level of the antiapoptotic factor GRP78 was increased after 4-PBA treatment compared with emodin-treated lung cancer cells (Fig. 2C). Furthermore, the level of the pro-apoptotic factor CHOP was decreased after 4-PBA treatment compared with emodintreated A549 and H1299 cells (Fig. 2C).

Emodin-induced apoptosis of lung cancer cell is TRIB3 dependent. ER stress in particular was previously shown to trigger TRIB3 signaling activation (14) and TRIB3 has been demonstrated to be important in the regulation of cell apoptosis (12), the current study aimed to clarify whether TRIB3 regulated emodin-induced apoptosis of A549 and H1299 cells. siRNA was used to knock down TRIB3 expression in lung cancer cells (Fig. 3A) and cell apoptosis was then evaluated by Hoechst staining. As shown in Fig. 3B and C, downregulation of TRIB3 significantly inhibited cancer cell apoptosis induced by emodin. Furthermore, emodin also increased the expression of TRIB3; and this effect was inhibited by pretreatment with 4-PBA, which inhibits ER stress (Fig. 3D).

Apoptosis of lung cancer cells caused by emodin-induced ER stress is associated with the TRIB3/NF- $\kappa B$ pathway. A previous study reported that TRIB3 altered ER stress-induced cell apoptosis via the NF- $\mathrm{KB}$ pathway (15). In order to investigate the potential mechanism involved in the anticancer effects of emodin, the current study focused on the ER stress/ TRIB3/NF-kB pathway in A549 and H1299 cells treated with emodin. To determine the effect of NF- $\mathrm{KB}$ inhibition during 


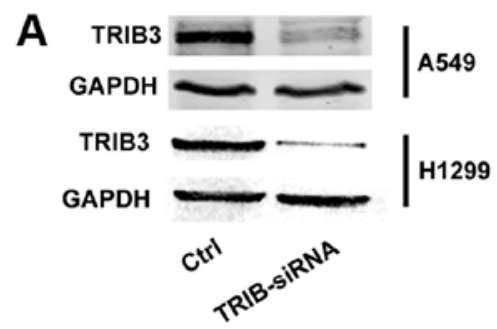

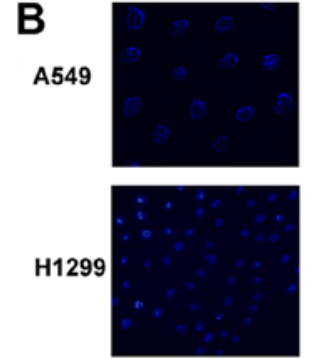

Ctrl
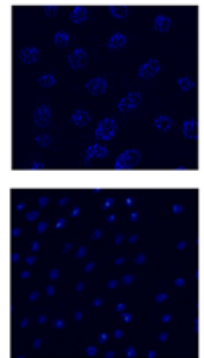

Ctrl+TRIB-3*
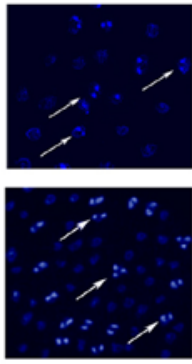

Emodin
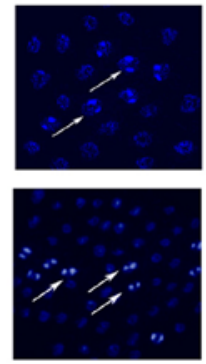

Emodin+TRIB3*
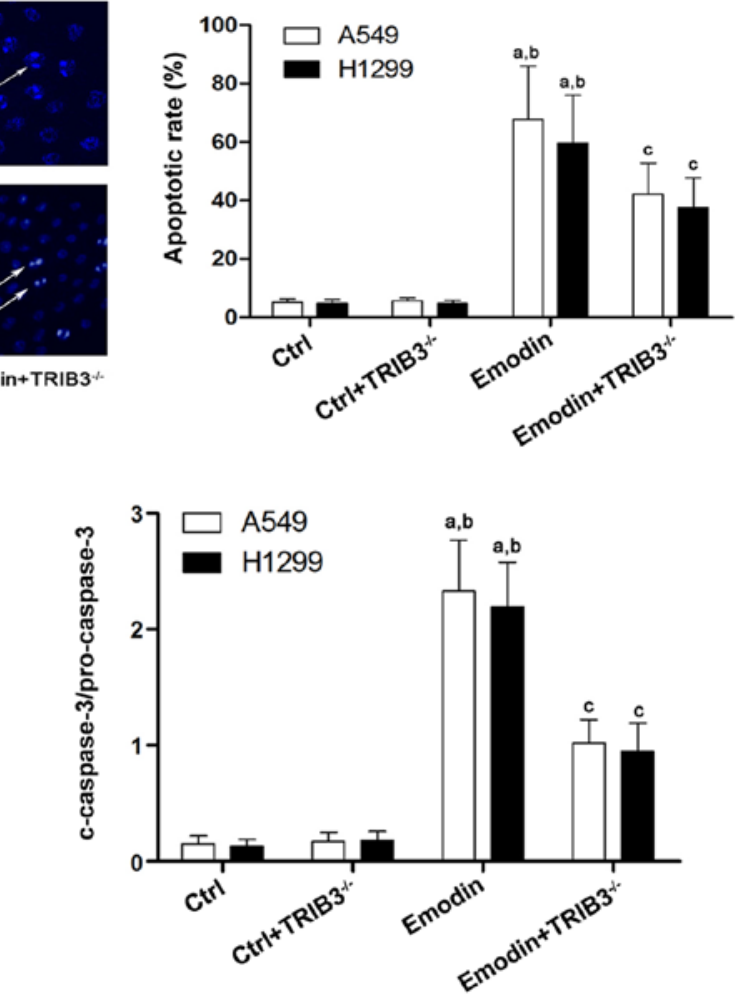

D

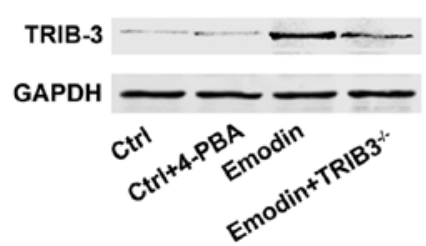

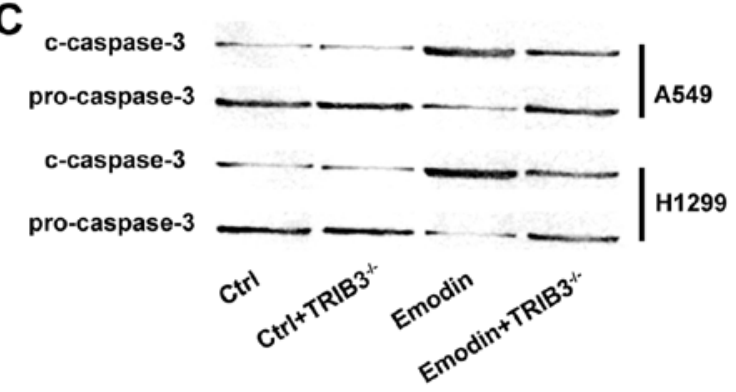

H1299
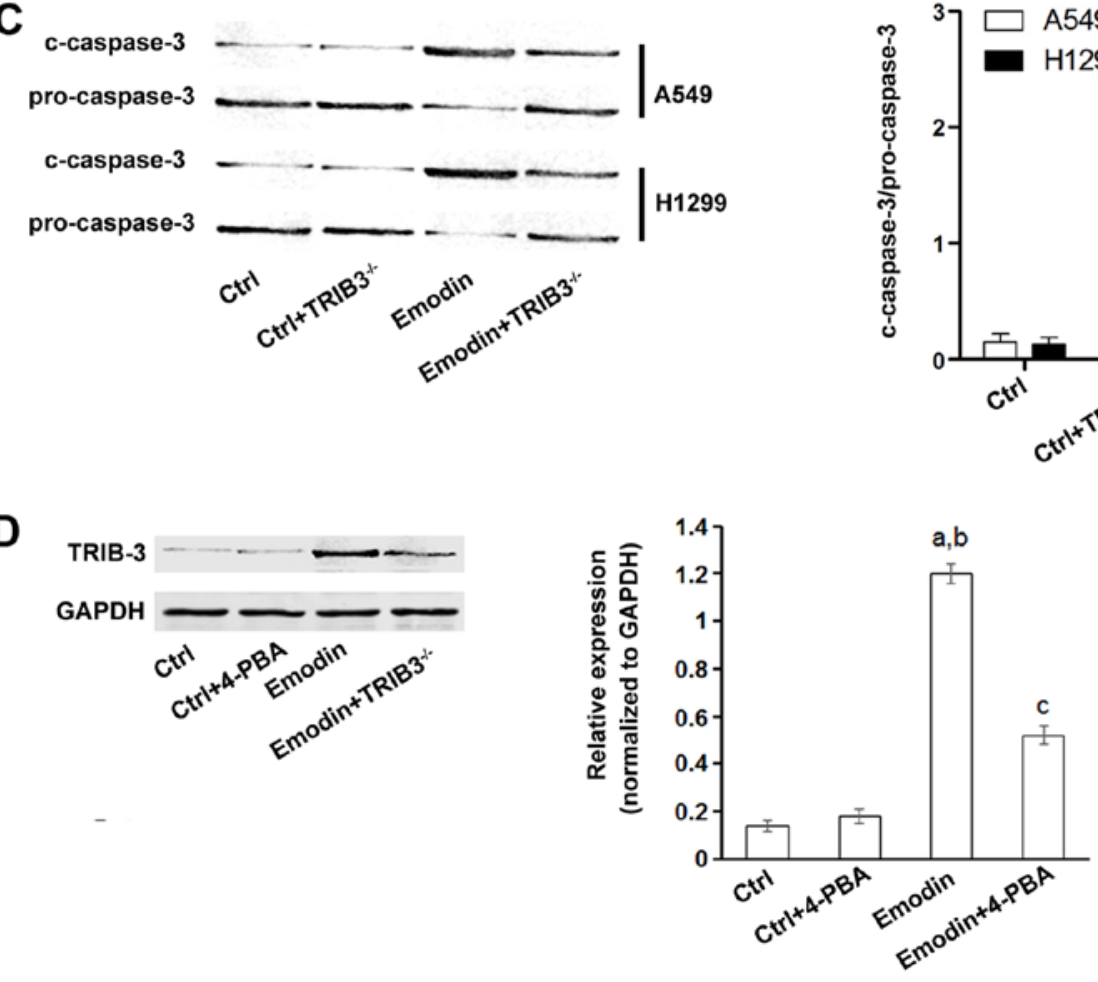

Figure 3. Emodin-induced apoptosis of lung cancer cells is TRIB3-dependent. (A) Reduced TRIB3 siRNA-knockdown in A549 cells was detected by western blot analysis. Hoechst fluorescent staining (B) and caspase-3 activation assay (C) were used to test the effect of downregulation of TRIB3 on cell apoptosis induced by emodin $(80 \mu \mathrm{mol} / 1)$ in A549 and $\mathrm{H} 1299$ cells $\left({ }^{\mathrm{a}} \mathrm{P}<0.05\right.$ vs. $\mathrm{Ctrl} ;{ }^{b} \mathrm{P}<0.05$ vs. Ctrl + TRIB3 ${ }^{-1}$; ${ }^{\circ} \mathrm{P}<0.05$ vs. emodin) is shown. (D) A549 cells were treated with emodin $(80 \mu \mathrm{mol} / \mathrm{l})$ with or without incubation with 4-PBA $(500 \mu \mathrm{mol} / \mathrm{l})$ for $72 \mathrm{~h}$. Western blot analysis was used to determine the expression of TRIB3. Columns demonstrate the relative expression levels of TRIB3 normalized to GAPDH. The data are presented as the mean \pm standard deviation from three independent experiments ( ${ }^{\mathrm{P}} \mathrm{P}<0.05 \mathrm{vs}$. Ctrl; ${ }^{\mathrm{b}} \mathrm{P}<0.05 \mathrm{vs}$. Ctrl + 4-PBA; ${ }^{\mathrm{P}} \mathrm{P}<0.05$ vs. emodin). siRNA, small interfering RNA; TRIB3, tribbles homolog 3; Ctrl, control; 4-PBA, 4-phenylbutyrate.

emodin-induced A549 and H1299 cell apoptosis, a selective inhibitor of NF- $\kappa B$, PDTC (Sigma-Aldrich; Merck Millipore), was used. As shown in Fig. 4A, emodin failed to increase apoptosis in lung cancer cells after the cells were exposed to PDTC. Furthermore, treatment with emodin increased NF- $\kappa B$ translocation from the cytoplasm to the nucleus and downregulation of TRIB3 inhibited this effect (Fig. 4B), indicating that TRIB3/NF- $\kappa B$ pathway is involved in emodin-induced lung cancer apoptosis.

Emodin inhibits subcutaneous tumors generated by inducing ER stress-dependent apoptosis in vivo. To investigate the in vivo actions of emodin on tumor development, subcutaneous tumors were generated by implanting A549 cells in 

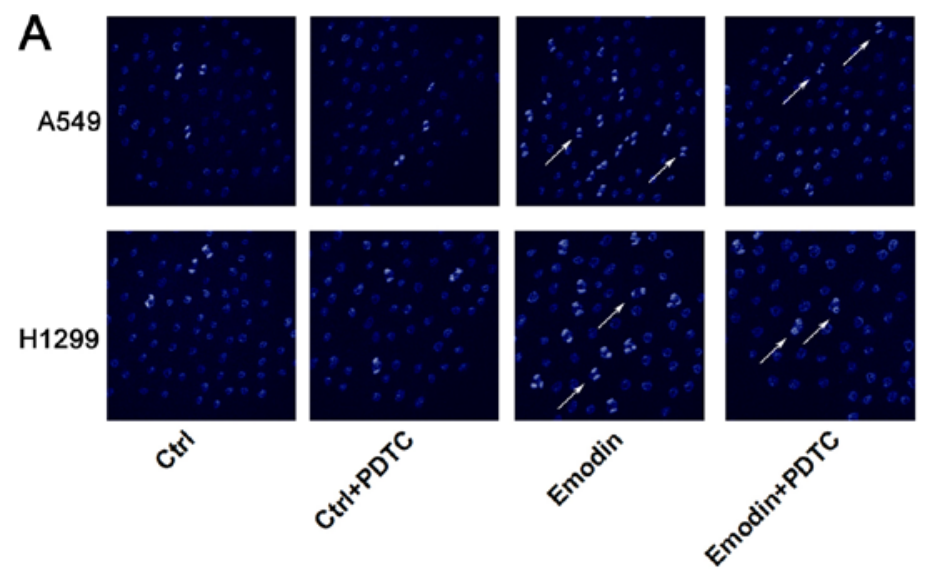

s

B

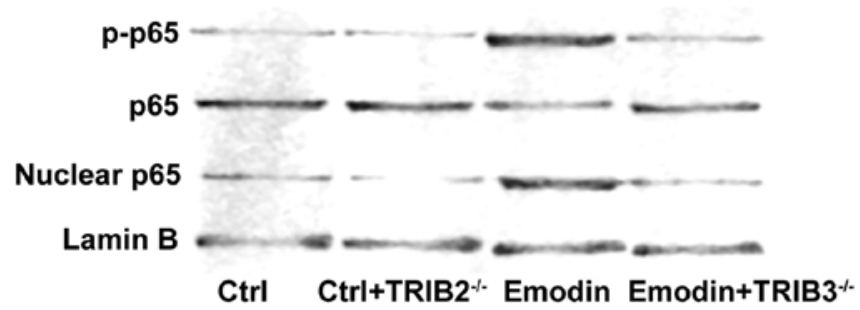

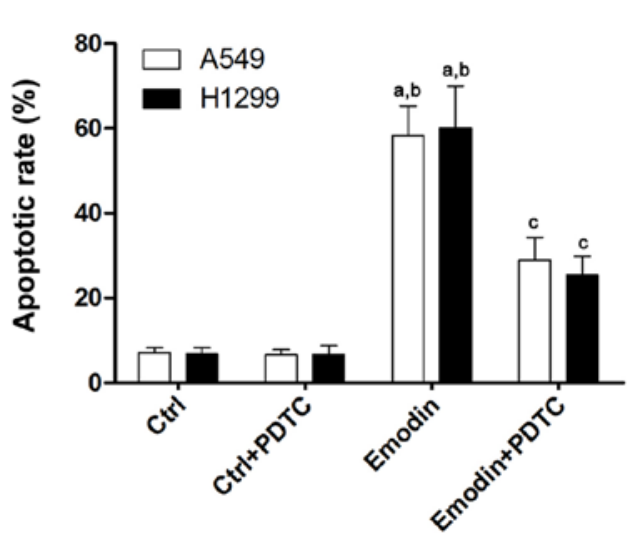

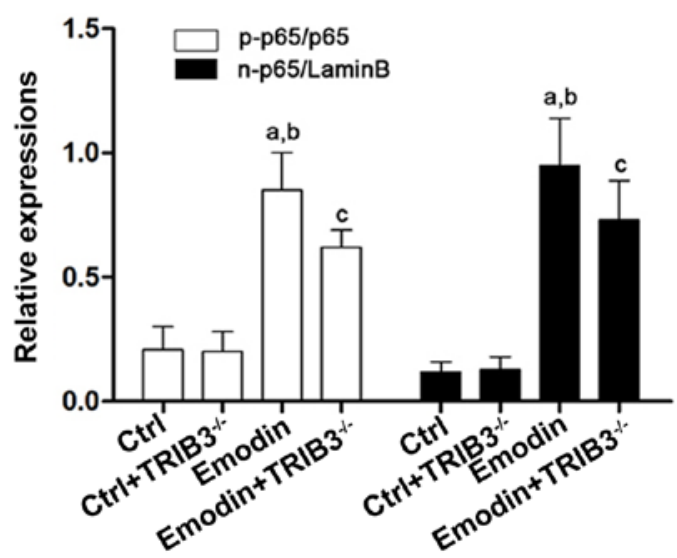

Figure 4. ER stress-induced apoptosis of A549 cells caused by emodin is associated with TRIB3/NF- $\kappa$ B. A549 and H1299 cells were treated with emodin

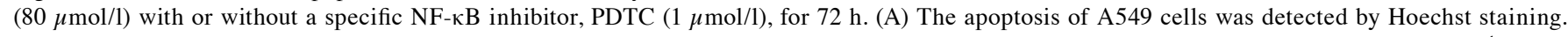
Columns demonstrate the apoptotic rates of A549 an $\mathrm{H} 1299$ cells in the Ctrl, Ctrl + PDTC, emodin, and emodin + PTDC groups ( ${ }^{\mathrm{a}} \mathrm{P}<0.05$ vs. Ctrl; ${ }^{b} \mathrm{P}<0.05$ vs. Ctrl + PDTC; ${ }^{c} \mathrm{P}<0.05$ vs. emodin). (B) Western blotting was used to detect p-p65, p65, nuclear p65 and lamin B in the Ctrl, Ctrl+TRIB3 ${ }^{-/}$, emodin, and emodin + TRIB3 $^{-/}$groups of A549 cells. The quantitation data of western blots are presented as the mean \pm standard deviation from three independent experiments ( ${ }^{\mathrm{a}} \mathrm{P}<0.05$ vs. Ctrl; ${ }^{\mathrm{b}} \mathrm{P}<0.05$ vs. Ctrl + TRIB3 ${ }^{-/}$; ${ }^{\mathrm{c}} \mathrm{P}<0.05$ vs. emodin). ER, endoplasmic reticulum; PDTC, pyrrolidinedithiocarbamate; Ctrl, control; TRIB, tribbles homolog $3 ; \mathrm{NF}-\kappa \mathrm{B}$, nuclear factor- $\kappa \mathrm{B}$.

$\mathrm{BALB} / \mathrm{c}$ nu/nu nude mice. Supporting the in vitro results, tumors treated with emodin grew more slowly than control tumors (Fig. 5A). However, the inhibitory effects of emodin on tumor growth were significantly reduced by co-treatment with 4-PBA (Fig. 5A). At 40 days after tumor initiation, tumors were removed and macroscopically measured. As shown in Fig. 5B, emodin significantly reduced tumor volume and this effect was inhibited by co-treatment with 4-PBA.

\section{Discussion}

The results of the current study, which demonstrated the inhibitory effects of emodin on NSCLC cell viability, were in accordance with previous studies (21). The results also confirmed that emodin reduced lung cancer cell viability by inducing apoptosis. By detecting an ER stress marker, GRP78, and an ER stress pro-apoptotic factor, CHOP, we demonstrated that the apoptosis-inducing effect of emodin on A549 and H1299 cells was mediated by ER stress. In order to identify a specific molecular target of emodin, the TRIB3/NF- $\kappa \mathrm{B}$ signaling pathway was investigated. The results revealed that emodin did affect TRIB3 expression, which may induce cell apoptosis in emodin-treated A549 and H1299 cells.
As one of the most basic and important organelles of mammalian cells, the ER executes multiple vital biological functions, including protein folding, protein maturation, protein post-translational modification, calcium signaling and lipid synthesis to maintain intracellular homeostasis (22). When a cell encounters harmful stimuli, ER stress is triggered and initially presents as the unfolded protein response (UPR) (23). UPR eliminates damaged proteins and initiates global inhibition of protein transcription (24). However, when the stimulus is prolonged and aggravated, the apoptotic signals are transduced by ER stress pathways to destroy damaged cells (25). GRP78 and CHOP are generally accepted as molecular markers of ER stress. Furthermore, CHOP is recognized as a pro-apoptotic factor during ER stress (26).

In the present study, incubation with emodin reduced the viability of A549 and H1299 cells in a concentrationdependent manner. Apoptosis analysis also demonstrated that emodin induced apoptosis of lung cancer cells in a concentration-dependent manner. These results were in accordance with previous studies. Our results suggested that the apoptotic avidity of emodin was mediated by ER stress, as demonstrated by the observation that an ER stress inhibitor, 4-PBA, reduced the expression levels of GRP78 

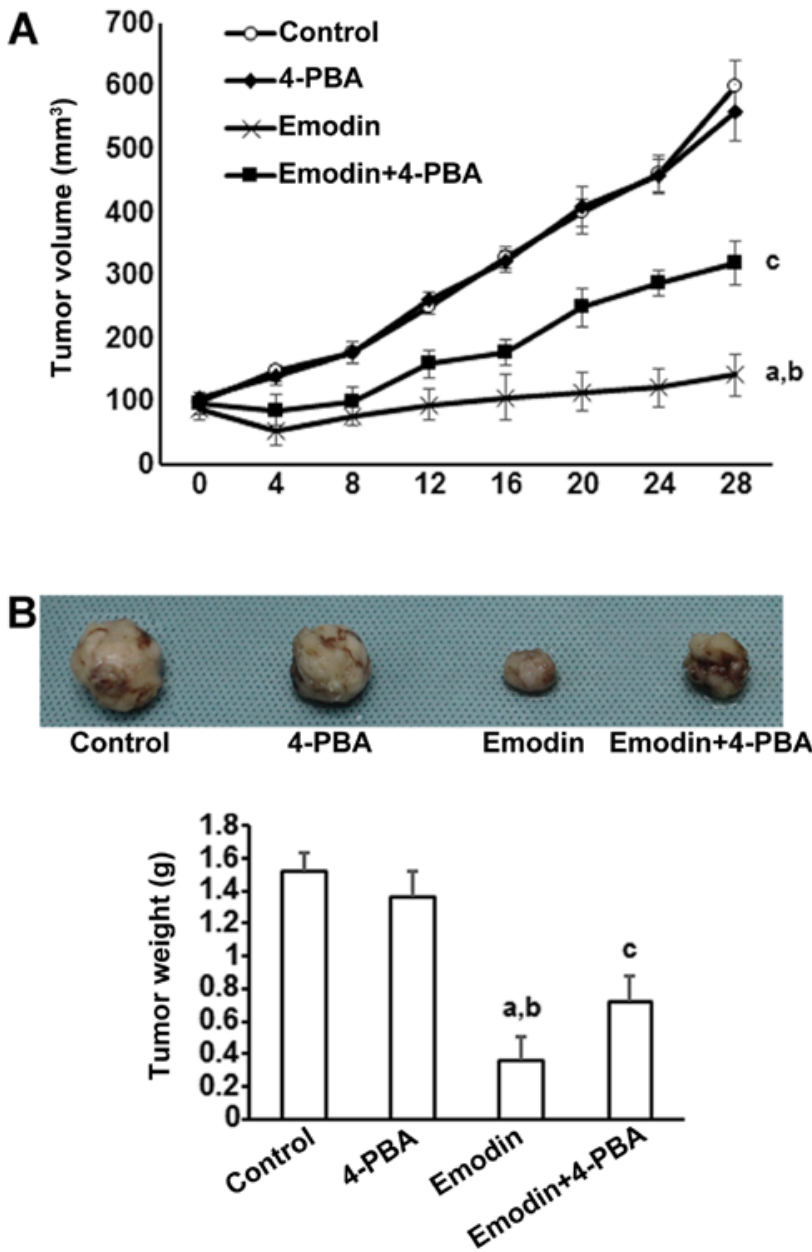

Figure 5. Emodin inhibits subcutaneous tumors generated by inducing ER stress-dependent apoptosis in vivo. (A) Nude mice were subcutaneously injected with $2.5 \times 10^{6}$ A549 cells and treated with: i) Ctrl; ii) $20 \mathrm{mg} / \mathrm{kg} / \mathrm{day}$ 4-PBA alone; iii) $50 \mathrm{mg} / \mathrm{kg} / \mathrm{day}$ emodin; and iv) $50 \mathrm{mg} / \mathrm{kg} /$ day emodin combined with $20 \mathrm{mg} / \mathrm{kg} / \mathrm{day} 4-\mathrm{PBA}$. Tumor volume was recorded and presented as the mean \pm standard deviation. Each group was composed of 8 mice (B) Representative images of the tumors from mice 40 days after implantation and a quantitative summary of the tumor weights are shown $\left({ }^{\mathrm{a}} \mathrm{P}<0.05\right.$ vs. Ctrl; ${ }^{\mathrm{b}} \mathrm{P}<0.05$ vs. $\mathrm{Ctrl}+4-\mathrm{PBA} ;{ }^{\mathrm{c}} \mathrm{P}<0.05$ vs. emodin). $\mathrm{ER}$, endoplasmic reticulum; 4-PBA, 4-phenylbutyrate.

and CHOP, and also impaired emodin-induced apoptosis in lung cancer cells.

TRIB3 is a mammalian homolog of the protein tribbles in Drosophila which participates in various cellular processes, such as migration and mitosis. Several recent studies reported that cancer prognosis and TRIB3 levels are closely correlated (16). It was reported that cancer patients with higher levels of TRIB3 protein had better prognosis compared with patients with low TRIB3 levels (13). These results indicated that TRIB3 was functionally associated with malignant cancer. ER stress is activated by stimuli, and apoptotic signaling following ER stress is transduced through an ATF4/CHOP pathway following phosphorylation of eukaryotic initiation factor $2 \alpha$. A previous study reported that TRIB3 expression was induced by ER stress via the ATF4/CHOP pathway as the promoter region of TRIB3 is an ER stress response element with a CHOP binding site (27). In the present study, the TRIB3 expression level was elevated by emodin-induced ER stress.
In this study, emodin treatment of A549 and H1299 cells triggered ER stress-mediated apoptosis. When ER stress was suppressed by 4-PBA, the expression levels of CHOP/TRIB3 were also reduced. As a result, emodin-induced lung cancer cell apoptosis was also inhibited. Furthermore, TRIB3 silencing using siRNA impaired emodin-induced ER stressmediated apoptosis, even though ER stress was not inhibited in lung cancer cells. These results indicated TRIB3 may be a molecular target of emodin when inducing apoptosis via ER stress.

Previous studies reported that TRIB3 activates NF- $\kappa \mathrm{B}$ activity by directly binding to $\mathrm{p} 65$, promoting its nuclear translocation and phosphorylation (15), which would promote cell death due to the increased transcription of target genes, including cytokines (such as tumor necrosis factor) and c-Jun $\mathrm{N}$-terminal kinase, which triggers the caspase cascade (28). Additionally, we found that when ER stress was activated by emodin, TRIB3/NF- $\mathrm{KB}$ signaling was also activated. However, when ER stress was repressed or TRIB3 was silenced, NF- $\mathrm{kB}$ signaling was also inhibited, reducing the pro-apoptotic effects of emodin.

In conclusion, the results of the current study suggested that the potent anticancer effects of emodin are caused by ER stress-mediated apoptosis in lung cancer cells. We also provided evidence that TRIB3 is one of the important molecules involved in mediating emodin-induced apoptosis, and that TRIB3/NF- $\mathrm{kB}$ signaling participated in this process. Together, these findings improve the understanding of the mechanisms of the anticancer activity of emodin against lung cancer, and provide a theoretical basis for the clinical application of novel emodin-based anticancer agents in the future.

\section{Acknowledgements}

This study was supported by grant from the National Natural Science Foundation of China (no. 81502295).

\section{References}

1. Yin QW, Sun XF, Yang GT, Li XB, Wu MS and Zhao J: Increased expression of microRNA-150 is associated with poor prognosis in non-small cell lung cancer. Int J Clin Exp Pathol 8: 842-846, 2015.

2. Wang Z, Fu J, Diao D and Dang C: Pre-operative plasma D-dimer level may predict the poor prognosis within one year after the surgery for non-small cell lung cancer. Zhongguo Fei Ai Za Zhi 14: 534-537, 2011 (In Chinese).

3. Cooper S and Spiro SG: Small cell lung cancer: Treatment review. Respirology 11: 241-248, 2006.

4. Arumuggam N, Bhowmick NA and Rupasinghe HP: A review: phytochemicals targeting JAK/STAT signaling and IDO expression in cancer. Phytother Res 29: 805-817, 2015.

5. He L, Bi JJ, Guo Q, Yu Y and Ye XF: Effects of emodin extracted from Chinese herbs on proliferation of non-small cell lung cancer and underlying mechanisms. Asian Pac J Cancer Prev 13: 1505-1510, 2012.

6. Yu JQ, Bao W and Lei JC: Emodin regulates apoptotic pathway in human liver cancer cells. Phytother Res 27: 251-257, 2013.

7. Li XX, Dong Y, Wang W, Wang HL, Chen YY, Shi GY, Yi J and Wang J: Emodin as an effective agent in targeting cancer stem-like side population cells of gallbladder carcinoma. Stem Cells Dev 22: 554-566, 2013.

8. Yaoxian W, Hui Y, Yunyan Z, Yanqin L, Xin G and Xiaoke W: Emodin induces apoptosis of human cervical cancer hela cells via intrinsic mitochondrial and extrinsic death receptor pathway. Cancer Cell Int 13: 71, 2013. 
9. Chun-Guang W, Jun-Qing Y, Bei-Zhong L, Dan-Ting J, Chong W, Liang Z, Dan Z and Yan W: Anti-tumor activity of emodin against human chronic myelocytic leukemia K562 cell lines in vitro and in vivo. Eur J Pharmacol 627: 33-41, 2010.

10. Ok S, Kim SM, Kim C, Nam D, Shim BS, Kim SH, Ahn KS Choi SH and Ahn KS: Emodin inhibits invasion and migration of prostate and lung cancer cells by downregulating the expression of chemokine receptor CXCR4. Immunopharmacol Immunotoxicol 34: 768-778, 2012.

11. Prudente S, Sesti G, Pandolfi A, Andreozzi F, Consoli A and Trischitta V: The mammalian tribbles homolog TRIB3, glucose homeostasis, and cardiovascular diseases. Endocr Rev 33: 526-546, 2012.

12. Fontanesi L, Colombo M, Scotti E, Buttazzoni L, Bertolini F, Dall'Olio S, Davoli R and Russo V: The porcine tribbles homolog 3 (TRIB3) gene: Identification of a missense mutation and association analysis with meat quality and production traits in Italian heavy pigs. Meat Sci 86: 808-813, 2010.

13. Wennemers M, Bussink J, Grebenchtchikov N, Sweep FC and Span PN: TRIB3 protein denotes a good prognosis in breast cancer patients and is associated with hypoxia sensitivity. Radiother Oncol 101: 198-202, 2011.

14. Nicoletti-Carvalho JE, Nogueira TC, Gorjão R, Bromati CR, Yamanaka TS, Boschero AC, Velloso LA, Curi R, Anhê GF and Bordin S: UPR-mediated TRIB3 expression correlates with reduced AKT phosphorylation and inability of interleukin 6 to overcome palmitate-induced apoptosis in RINm5F cells. J Endocrinol 206: 183-193, 2010.

15. Fang N, Zhang W, Xu S, Lin H, Wang Z, Liu H, Fang Q, Li C Peng $L$ and Lou J: TRIB3 alters endoplasmic reticulum stressinduced $\beta$-cell apoptosis via the NF-kB pathway. Metabolism 63: 822-830, 2014

16. Miyoshi N, Ishii H, Mimori K, Takatsuno Y, Kim H, Hirose H, Sekimoto M, Doki Y and Mori M: Abnormal expression of TRIB3 in colorectal cancer: A novel marker for prognosis. Br J Cancer 101: 1664-1670, 2009.

17. Huang X, Taeb S, Jahangiri S, Emmenegger U, Tran E, Bruce J, Mesci A, Korpela E, Vesprini D, Wong CS, et al: miRNA-95 mediates radioresistance in tumors by targeting the sphingolipid phosphatase SGPP1. Cancer Res 73: 6972-6986, 2013.

18. Liu Z, Lv Y, Zhao N, Guan G and Wang J: Protein kinase R-like ER kinase and its role in endoplasmic reticulum stress-decided cell fate. Cell Death Dis 6: e1822, 2015.
19. Leamy AK, Egnatchik RA, Shiota M, Ivanova PT, Myers DS, Brown HA and Young JD: Enhanced synthesis of saturated phospholipids is associated with ER stress and lipotoxicity in palmitate treated hepatic cells. J Lipid Res 55: 1478-1488, 2014.

20. Cho JA, Zhang X, Miller GM, Lencer WI and Nery FC: 4-Phenylbutyrate attenuates the ER stress response and cyclic AMP accumulation in DYT1 dystonia cell models. PLoS One One 9: e110086 2014

21. Wei W-T, Lin S-Z, Liu D-L and Wang Z-H: The distinct mechanisms of the antitumor activity of emodin in different types of cancer (Review). Oncol Rep 30: 2555-2562, 2013.

22. Pluquet O, Pourtier A and Abbadie C: The unfolded protein response and cellular senescence. A review in the theme: Cellular mechanisms of endoplasmic reticulum stress signaling in health and disease. Am J Physiol Cell Physiol 308: C415-C425, 2015.

23. Lakshmanan AP, Harima M, Suzuki K, Soetikno V, Nagata M, Nakamura T, Takahashi T, Sone H, Kawachi H and Watanabe K: The hyperglycemia stimulated myocardial endoplasmic reticulum (ER) stress contributes to diabetic cardiomyopathy in the transgenic non-obese type 2 diabetic rats: A differential role of unfolded protein response (UPR) signaling proteins. Int J Biochem Cell Biol 45: 438-447, 2013.

24. Vidal RL, Figueroa A, Court FA, Thielen P, Molina C, Wirth C, Caballero B, Kiffin R, Segura-Aguilar J, Cuervo AM, et al: Targeting the UPR transcription factor XBP1 protects against Huntington's disease through the regulation of FoxO1 and autophagy. Hum Mol Genet 21: 2245-2262, 2012.

25. Fribley AM, Miller JR, Reist TE, Callaghan MU and Kaufman RJ: Large-scale analysis of UPR-mediated apoptosis in human cells. Methods Enzymol 491: 57-71, 2011.

26. Li Y, Guo Y, Tang J, Jiang J and Chen Z: New insights into the roles of CHOP-induced apoptosis in ER stress. Acta Biochim Biophys Sin (Shanghai) 47: 146-147, 2015.

27. Bromati CR, Lellis-Santos C, Yamanaka TS, Nogueira TC, Leonelli M, Caperuto LC, Gorjão R, Leite AR, Anhê GF and Bordin S: UPR induces transient burst of apoptosis in islets of early lactating rats through reduced AKT phosphorylation via ATF4/CHOP stimulation of TRB3 expression. Am J Physiol Regul Integr Comp Physiol 300: R92-R100, 2011.

28. Zhang R, Cao X, Wang C, Hou L, Nie J, Zhou M and Feng Y: An antitumor peptide from Musca domestica pupae (MATP) induces apoptosis in HepG2 cells through a JNK-mediated and Akt-mediated NF-kB pathway. Anticancer Drugs 23: 827-835, 2012. 